\title{
Erratum to: Thermodynamic aspects of rock friction
}

\author{
N. Mitsui • P. Ván
}

Published online: 17 January 2015

(C) Akadémiai Kiadó 2015

\section{Erratum to: Acta Geod Geophys (2014) 49:135-146 DOI 10.1007/s40328-014-0048-6}

1. In Eq. (5) page 137 a minus sign is missing in the argument of the exponential, correctly it is $\Theta_{*}-\Theta$.

2. The formulas (30) and (31) and the regarding discussion about the conditions of velocity strengthening and weakening of steady-state friction is to be corrected.

Instead of (30) the condition of velocity strengthening is:

$$
\frac{\beta_{c}}{\left(1+\frac{\beta_{c}}{\alpha_{c}} V_{1}\right)\left(1+\frac{\beta_{c}}{\alpha_{c}} V_{2}\right)}>l_{12} \frac{l_{21}}{l_{2}} .
$$

Instead of (31) the condition of velocity weakening is:

$$
\frac{\beta_{c}}{\left(1+\frac{\beta_{c}}{\alpha_{c}} V_{1}\right)\left(1+\frac{\beta_{c}}{\alpha_{c}} V_{2}\right)}<l_{12} \frac{l_{21}}{l_{2}} \text {. }
$$

3. The lines 3-4 on page 143 "The sign of coefficient of $V_{s s}$ in Eq. (29) distinguishes between velocity strengthening and weakening."

should be replaced by

"We can derive the velocity dependency of steady-state friction by using Eq. (29)."

The online version of the original article can be found under doi:10.1007/s40328-014-0048-6.

N. Mitsui $(\bowtie) \cdot$ P. Ván

Department of Theoretical Physics, Wigner RCP, RMKI, P.O. Box 49, 1525 Budapest, Hungary

e-mail: mitsui.noa@wigner.mta.hu

P. Ván

Department of Energy Engineering, Budapest University of Technology and Economics,

Bertalan Lajos u. 4-6, Budapest 1111, Hungary

e-mail: van.peter@wigner.mta.hu 
4. On page 144 the lines $1-6$ should be replaced to the following sentences:

"Both velocity strengthening and weakening are compatible with the thermodynamic conditions (13), that is, they both satisfy the second law of thermodynamics. Furthermore, the differences in requirements between Eqs. (30) and (31) indicate a possible mechanism in this respect. In this model, it is caused by the difference between the linearity of the instantaneous jump and that of the following relaxation to the load-point velocity. A possible explanation of the mechanism can be the appearance of dissipative structures during frictional jump and the following relaxation." 